\title{
A Diabetogenic Polypeptide from Bovine Adenohypophysis Similar to that Excreted in Lipoatrophic Diabetes
}

\author{
By Lawrence H. Louis, Jerome W. Conn and Merlyn C. Minick
}

A polypeptide has been isolated from bovine adenohypophysis which antagonizes the hypoglycemic effect of exogenous insulin and which, per se, induces loss of carbohydrate tolerance in men and dogs. Mild acid hydrolysis of the active polypeptide yields a compound which retains the same biological properties. Characteristics of the active principle and its hydrolytic product is the long duration of their activities, the greatest intensity of the effects being observed between 34 and 60 hours after a single intramuscular injection. Both substances are devoid of ACTH activity. The active polypeptide resembles closely the insulin antagonist isolated from the urine of patients with lipoatrophic diabetes previously reported from this laboratory. Details of the isolation and physiologic effects of the active substance and its hydrolytic product are described. (Metabolism 15: No. 4, April, 308-324, 1966)

I RECENT COMMUNICATIONS from this laboratory, ${ }^{1,2}$ the isolation of an insulin antagonist from the urine of patients with lipoatrophic diabetes has been reported. It also was demonstrated that a similar substance was present in the urine of a maturity-onset insulin-resistant diabetic without lipoatrophy. The substance was found to be a polypeptide which when administered to either dogs or man exhibited diabetogenic and anti-insulin effects. The origin of the active principle had not been determined.

The present report describes a procedure for the isolation of a similar substance from the anterior lobes of bovine pituitary glands. Like the insulin antagonist from the urine, the material is also highly active both in dogs and in man.

\section{Materials and Methods}

Frozen bovine anterior pituitary glands, obtained from the Armour Pharmaceutical Company, Kankakee, Illinois, were treated according to the procedure shown in Figure 1. The

The Department of Internal Medicine (Division of Endocrinology and Metabolism ard the Metabolism Research Unil), the University of Michigan Medical School, Ann Arbor, Michigan.

Presented in part at the 25th Annual Meeting of the American Diabetes Association in New York City, June 20, 1965.

This work was supported by grant AM-06665-03 National Institute of Arthritis and Metabolic Diseases, United States Public Health Service.

Received for publication Nov. 22, 1965.

Lawrence H. Louts, Sc.D.: Associate Professor of Biological Chemistry, Department of Internal Medicine (Division of Endocrinology and Metabolism and the Metabolism Research Unit), the University of Michigan, Ann Arbor, Mich. Jenome W. ConN, M.D.: Professor of Internal Medicine, (Division of Endocrinology and Metabolism and the Metabolism Research Unit), the University of Michigan, Ann Arbor, Mich. MERIYN C. Minicx, M.S.: Research Associate, Department of Internal Medicine (Division of Endocrinology and Metabolistn and the Metabolism Research Unit), the University of Michigan, Ann Arbor, Mich. 


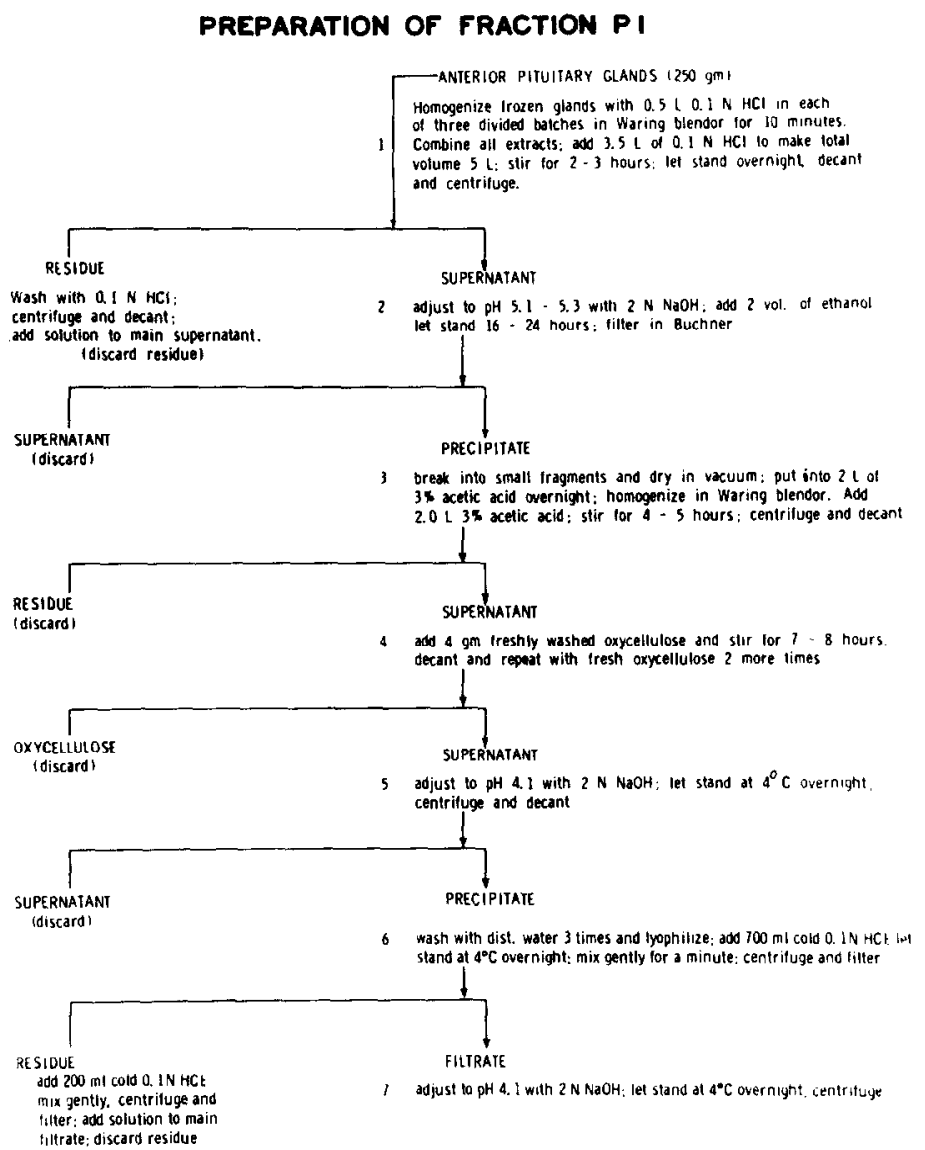

Fig. 1A

oxycellulose powder (17-21 per cent COOH, Eastman Chemical Products, Inc., Kingsport, Tennessee) employed in step 4 was kept refrigerated and before use was washed successively with distilled water, 0.1 N HCL, and distilled water. Electrophoresis of fraction PI on cellulose acetate reveals 3 bands, a prominent one with minor bands before and after. Elemental analysis of the compound gives the following; $\mathrm{C} 48.32$ per cent, $\mathrm{H} 7.18$ per cent, $\mathrm{N} 15.36$ per cent, and $\mathrm{S} 1.41$ per cent. Its isoelectric point is approximately $\mathrm{pH} 4.1$, and the average yield is $0.05-0.06$ per cent.

Dogs were maintained on a constant high carbohydrate diet $(454 \mathrm{Gm}$. of Pard and 75 $\mathrm{Gm}$. of glucose) and the human subjects on a constant 3,200 calorie diet including $300 \mathrm{Gm}$. of carbohydrate before and during all testing procedures. Glucose and insulin tolerance tests were carried out before and at various intervals after administration of the material. Twentyfour hour urine specimens were collected daily on the human subjects for the estimation of 17ketosteroids, ${ }^{3}$ 17-hydroxysteroids, ${ }^{4}$ uric acid, ${ }^{5}$ glucose, ${ }^{6}$ and creatinine. ${ }^{7}$ The active principle from the pituitary tissue was prepared for injection in exactly the same way as that previously described ${ }^{2}$ for the insulin antagonist extracted from urine.

\section{Results}

Fraction PI was tested for biological activity on 3 normal dogs. Figures 2 and 3 depict examples of the effects obtained on glucose tolerance and on insulin tolerance in dogs. 




Fig. 1B

Fraction PI also induces glucose intolerance and insulin resistance in man. Tables 1, 2 and 3 show the results obtained when $20 \mathrm{mg}$. was administered intramuscularly in a single dose to each of 3 normal volunteers. In each case the largest degree of carbohydrate intolerance was observed 8 hours following administration of PI. Table 4 shows that urinary excretion of 17-hydroxysteroids, 17-ketosteroids and uric acid did not change significantly.

Insulin tolerance tests were performed on 2 more healthy subjects. This time doses of 20 and $30 \mathrm{mg}$. of fraction PI were administered intramuscularly on 2 successive days and the insulin tolerance tests were done 9 and 33 hours after the second injection. Figures 4 and 5 indicate the marked insulin resistance which was induced. No untoward reacting to this material was observed in any subject.

Further Fractionation of Fraction PI. Biological Effects of Fraction PIIIC2

Figure 6 describes the procedure by which fraction PIIIC2 is obtained from 


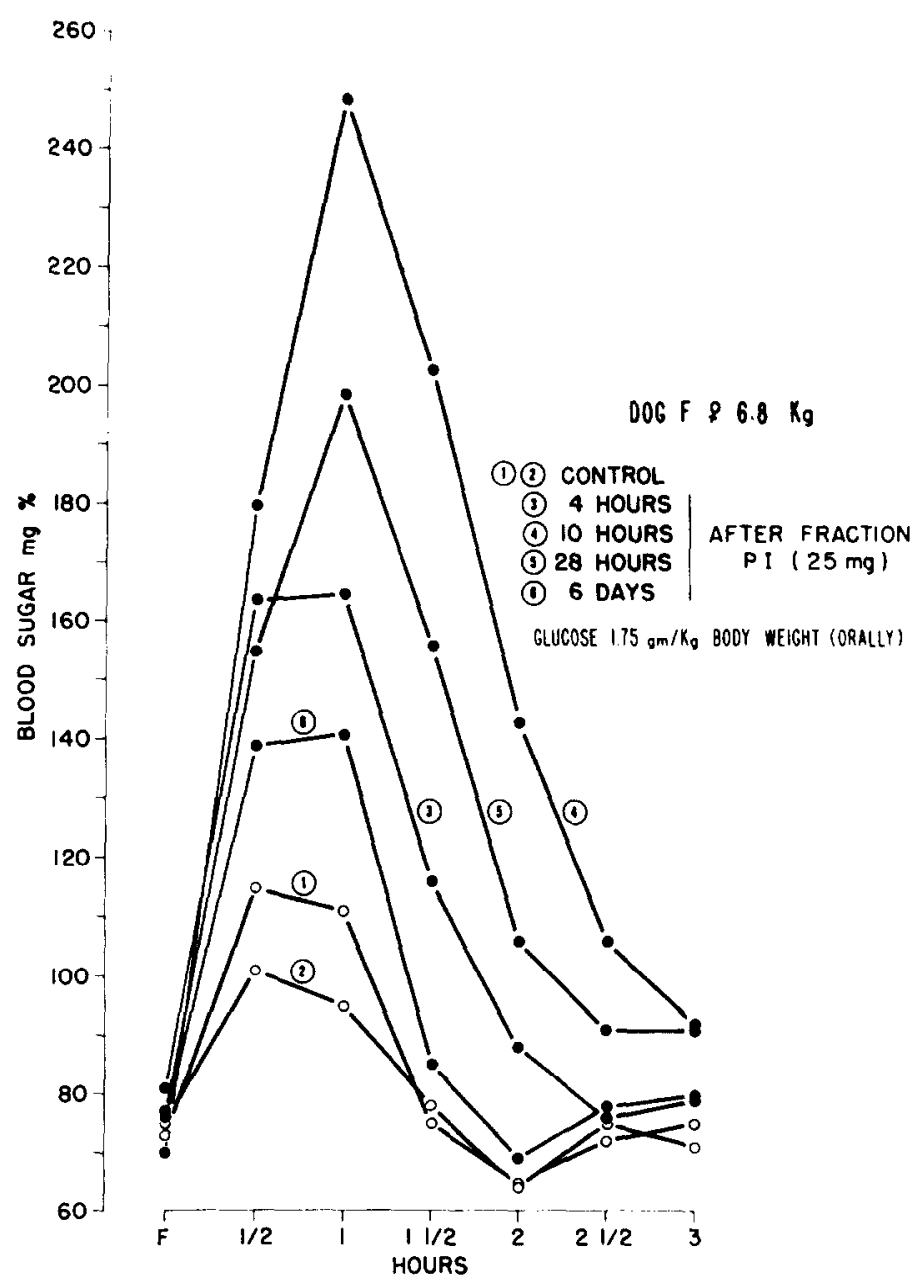

Fig. 2

fraction PI. Elemental analysis of this compound shows C 51.22 per cent, $\mathrm{H}$ 7.48 per cent, $\mathrm{N} 14.66$ per cent, and S 1.91 per cent. Its isoelectric point is the same (approximately pH 4.7 ) as the urinary fraction IIIC2.

Although fraction PIIIC2 is a hydrolytic product of fraction PI, it possesses diabetogenic and anti-insulin properties. However, in one set of experiments on the same $\operatorname{dog}$ (Fig. 7, 8 and 9), fraction PI was found to be approximately twice as potent as fraction PIIIC2.

Biological activity of fraction PIIIC2 was tested on 7 normal young men. Three of them received a single dose of 11-12 mg. intramuscularly and all developed glucose intolerance. Figures 10 and 11 show the results of subjects W. M. B. and J. S. S. Table 5 indicates that urinary excretion of 17 -hydroxysteroids, 17-ketosteroids, and uric acid did not change significantly.

Each of the other 4 subjects was given a larger single dose $(40 \mathrm{mg}$.) of the 


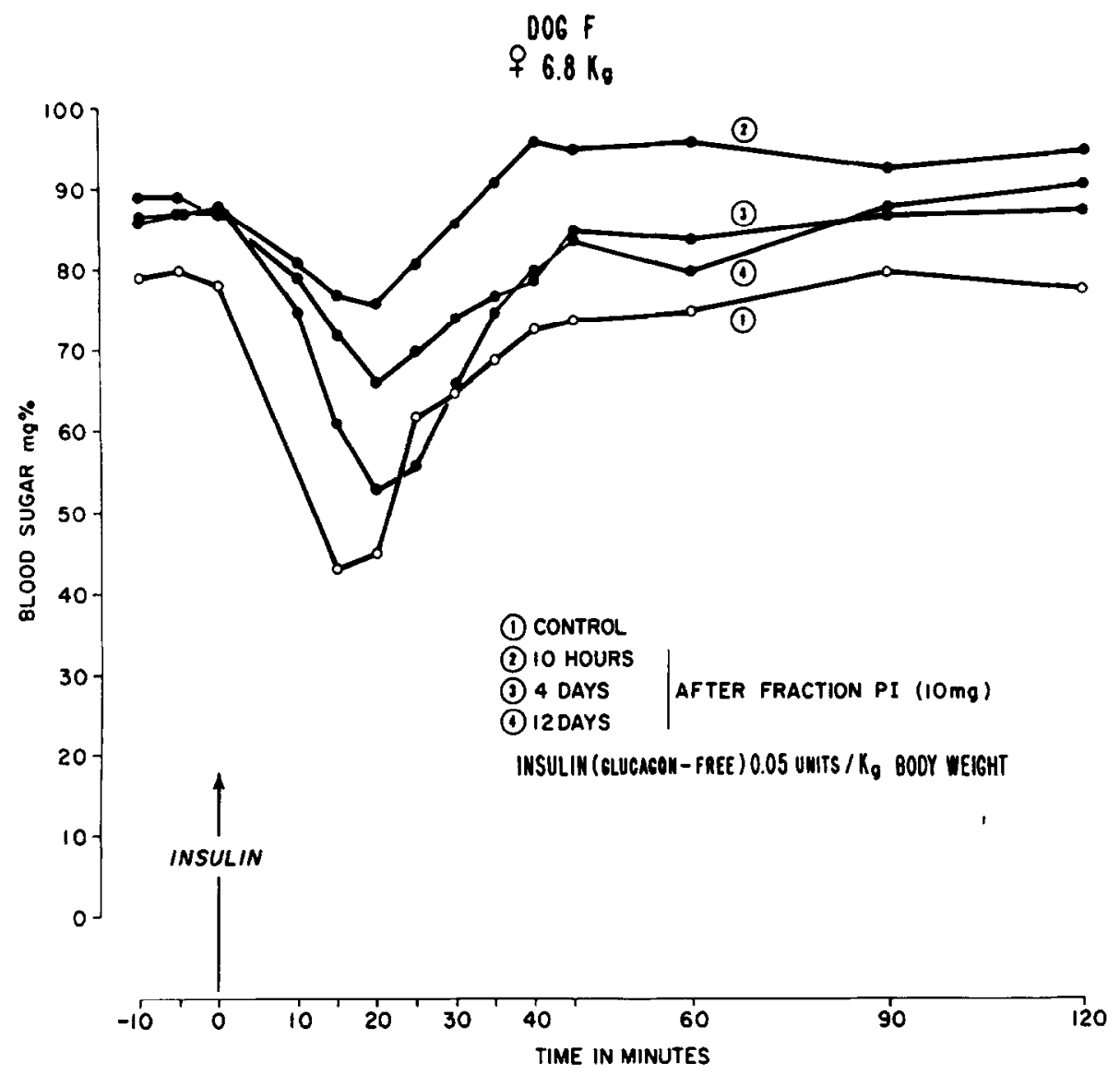

Fig. 3

same fraction. Glucose tolerance tests were performed daily for from 7 to 9 days. Tables 6, 7, 8 and 9 disclose the following: (1) the material induces carbohydrate intolerance which persists for 2 to 3 days; (2) renal glycosuria becomes evident; and (3) a resurgence of both (1) and (2) at about the sixth postinjection day.

A series of insulin tolerance tests were performed on W. M. B. following a $12 \mathrm{mg}$. dose of fraction PIIIC2, the results of which are shown on Figure 12.

\section{Discussion}

A diabetogenic polypeptide has been isolated from bovine adenohypophsis by the same procedure with which a similar diabetogenic polypeptide was previously isolated from the urine of patients with lipoatrophic diabetes. ${ }^{2}$ The physical properties of both compounds are very similar and have isoelectric points at approximately $\mathrm{pH}$ 4.1. Mild acid hydrolysis of both polypeptides yields a similar active substance which in both cases has an isoelectric point at approximately $\mathrm{pH} 4.7$. 
Table 1.-Effect of Fraction PI upon Glucose Tolerance on a Normal Subject J. C. M. (22 years, male, $75 \mathrm{Kg}$.)

\begin{tabular}{|c|c|c|c|c|c|c|c|}
\hline $\begin{array}{l}\text { HOURS AFTER } \\
\text { INJECTION } 20 \mathrm{mg}\end{array}$ & $F$ & $1 / 2$ & 1 & $11 / 2$ & 2 & $21 / 2$ & 3 \\
\hline Control & 81 & 129 & 98 & 85 & 82 & 98 & 87 \\
\hline 8 & 84 & 174 & $\begin{array}{c}160 \\
\text { (trace) }\end{array}$ & 127 & $\begin{array}{c}119 \\
\text { (trace) }\end{array}$ & 116 & 66 \\
\hline 33 & 96 & 134 & 151 & 118 & 104 & 87 & 103 \\
\hline 56 & 79 & 145 & 170 & 114 & 119 & 112 & 104 \\
\hline 81 & 75 & 126 & 133 & 105 & 105 & 105 & 76 \\
\hline 105 & 79 & 118 & 128 & 130 & 122 & 116 & 113 \\
\hline 131 & 79 & 128 & $\begin{array}{c}135 \\
\text { (trace) }\end{array}$ & $\begin{array}{c}120 \\
\text { (trace) }\end{array}$ & $\begin{array}{c}104 \\
\text { (trace) }\end{array}$ & $\begin{array}{c}85 \\
\text { (trace) }\end{array}$ & $\begin{array}{c}94 \\
\text { (trace) }\end{array}$ \\
\hline 155 & 73 & 130 & $\begin{array}{c}130 \\
\text { (trace) }\end{array}$ & $\begin{array}{c}120 \\
\text { (trace) }\end{array}$ & 113 & 75 & 81 \\
\hline 179 & 74 & 155 & $\begin{array}{l}112 \\
1+1\end{array}$ & $\begin{array}{c}119 \\
\text { (trace) }\end{array}$ & $\begin{array}{c}96 \\
\text { (trace) }\end{array}$ & 85 & 95 \\
\hline$\stackrel{2}{2}$ & 84 & 117 & 147 & 123 & 103 & 107 & 78 \\
\hline
\end{tabular}

Table 2.-Effect of Fraction PI upon Glucose Tolerance on a Normal Subject J. C. M. (22 years, male, $75 \mathrm{Kg}$.)

\begin{tabular}{ccccccccc}
$\begin{array}{c}\text { HOURS AFTER } \\
\text { INJECTION 20 mg }\end{array}$ & $F$ & $1 / 2$ & 1 & $11 / 2$ & 2 & $21 / 2$ & 3 \\
\hline Control & 74 & 124 & 118 & 101 & 100 & 97 & 82 \\
8 & 85 & 147 & $\begin{array}{c}156 \\
\text { (trace) }\end{array}$ & $\begin{array}{c}156 \\
\text { (trace) }\end{array}$ & $\begin{array}{c}108 \\
\text { (trace) }\end{array}$ & $\begin{array}{c}106 \\
\text { (trace) }\end{array}$ & 89 \\
32 & 84 & 147 & $\begin{array}{c}159 \\
\text { (trace) }\end{array}$ & $\begin{array}{c}114 \\
\text { (trace) }\end{array}$ & 115 & 110 & 104 \\
55 & 79 & 123 & $\begin{array}{c}144 \\
\text { (trace) }\end{array}$ & $\begin{array}{c}104 \\
\text { (trace) }\end{array}$ & $\begin{array}{c}109 \\
\text { (trace) }\end{array}$ & 96 & 86 \\
(trace)
\end{tabular}


Table 3.-Effect of Fraction PI upon Glucose Tolerance on a Normal Subject L. J. B. (22 years, male, 68.3 Kg.)

\begin{tabular}{cccccccc}
\hline $\begin{array}{c}\text { Hours after injection } \\
20 \mathrm{mg}\end{array}$ & $F$ & $1 / 2$ & 1 & $11 / 2$ & 2 & $21 / 2$ & 3 \\
\hline Control & 81 & 167 & 145 & 130 & 78 & 93 & 90 \\
8 & 82 & 117 & 158 & 113 & 130 & 103 & 92 \\
34 & 85 & 154 & 150 & 132 & 123 & 120 & 115 \\
58 & 83 & 138 & 143 & 149 & 101 & 118 & 83 \\
104 & 77 & 139 & 135 & 115 & 115 & 89 & 71 \\
\hline
\end{tabular}

Table 4.-Effect of Fraction PI upon Urinary 17-OHCS, 17-KS, Creatinine, Uric Acid and Glucose

\begin{tabular}{|c|c|c|c|c|c|c|c|}
\hline SUBJECT & DAY & INJECTION & $\begin{array}{c}17-\mathrm{OHCS} \\
\mathrm{mg} / \mathrm{doy}\end{array}$ & $\begin{array}{l}17-\mathrm{kS} \\
\mathrm{mg} / \text { day }\end{array}$ & $\begin{array}{c}\text { CREATININE } \\
\mathrm{g} / \text { day }\end{array}$ & $\begin{array}{l}\text { URIC ACID } \\
g / \text { day }\end{array}$ & $\begin{array}{l}\text { GLUCOSE } \\
g / \text { day }\end{array}$ \\
\hline \multirow{10}{*}{ B.A.G. } & 1 & 0 & 7.2 & 22.8 & 2.59 & 0.940 & 1.324 \\
\hline & 2 & 0 & 6.2 & 22.8 & 2.37 & 0.919 & 1.254 \\
\hline & 3 & 0 & 7.6 & 22.8 & 2.50 & 0.932 & 1.324 \\
\hline & 4 & $20 \mathrm{mg}$ (IM) & 6.0 & 21.6 & 2.24 & 0.735 & 1.104 \\
\hline & 5 & 0 & 6.6 & 18.6 & 2.50 & 0.795 & 1.216 \\
\hline & 6 & 0 & 7.4 & 22.8 & 2.56 & 0.786 & 1.226 \\
\hline & $?$ & 0 & 7.0 & 19.6 & 2.49 & 0.778 & 1.188 \\
\hline & 8 & 0 & 8.2 & 19.2 & 2.47 & 0.701 & 1.150 \\
\hline & 9 & 0 & 6.6 & 18.0 & 2.52 & 0.769 & 1.234 \\
\hline & 10 & 0 & 8.6 & 21.4 & 2.56 & 0.791 & 1.274 \\
\hline \multirow{6}{*}{ J.C.M. } & 1 & 0 & 10.8 & 19.0 & 1.91 & 0.772 & 0.838 \\
\hline & 2 & 0 & 9.4 & 18.6 & 1.97 & 0.805 & 0.706 \\
\hline & 3 & $20 \mathrm{mg}(\mathrm{IM})$ & 11.6 & 22.0 & 2.00 & 0.805 & 0.926 \\
\hline & 4 & 0 & 9.8 & 19.2 & 2.01 & 0.793 & 0.890 \\
\hline & 5 & 0 & 10.2 & 22.4 & 2.01 & 0.739 & 0.926 \\
\hline & 6 & 0 & 9.4 & 19.2 & 2.12 & 0.759 & 0.890 \\
\hline
\end{tabular}




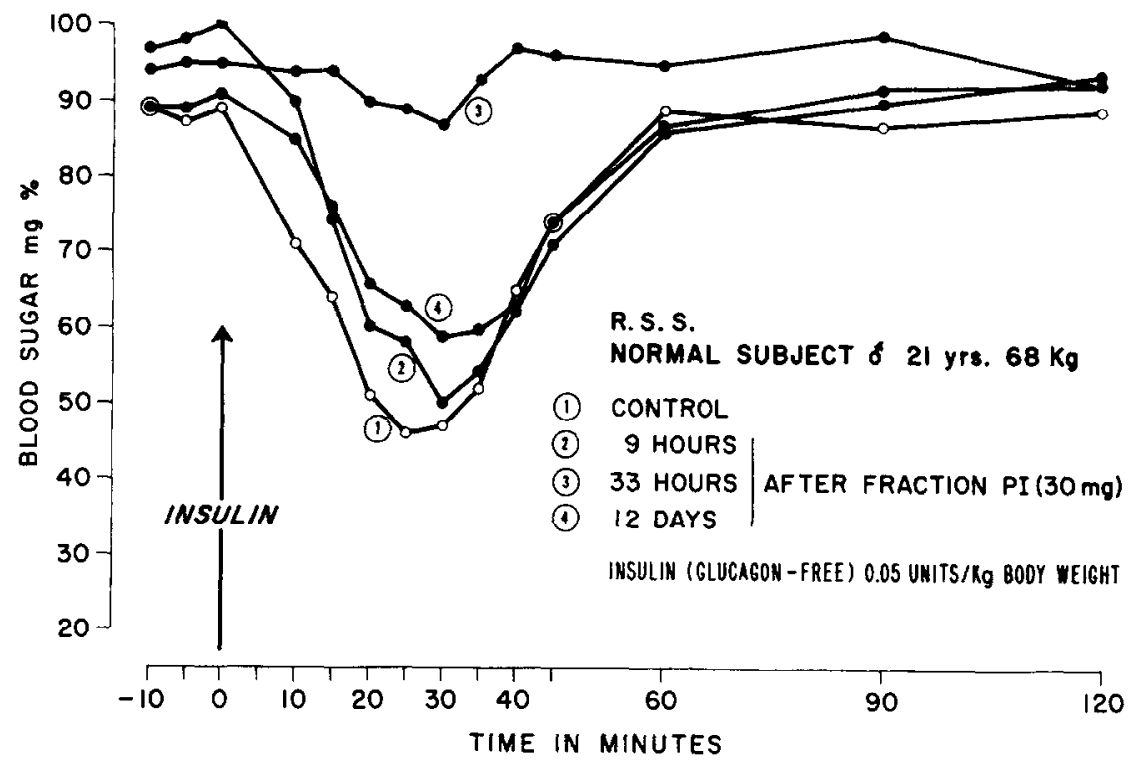

Fig. 4

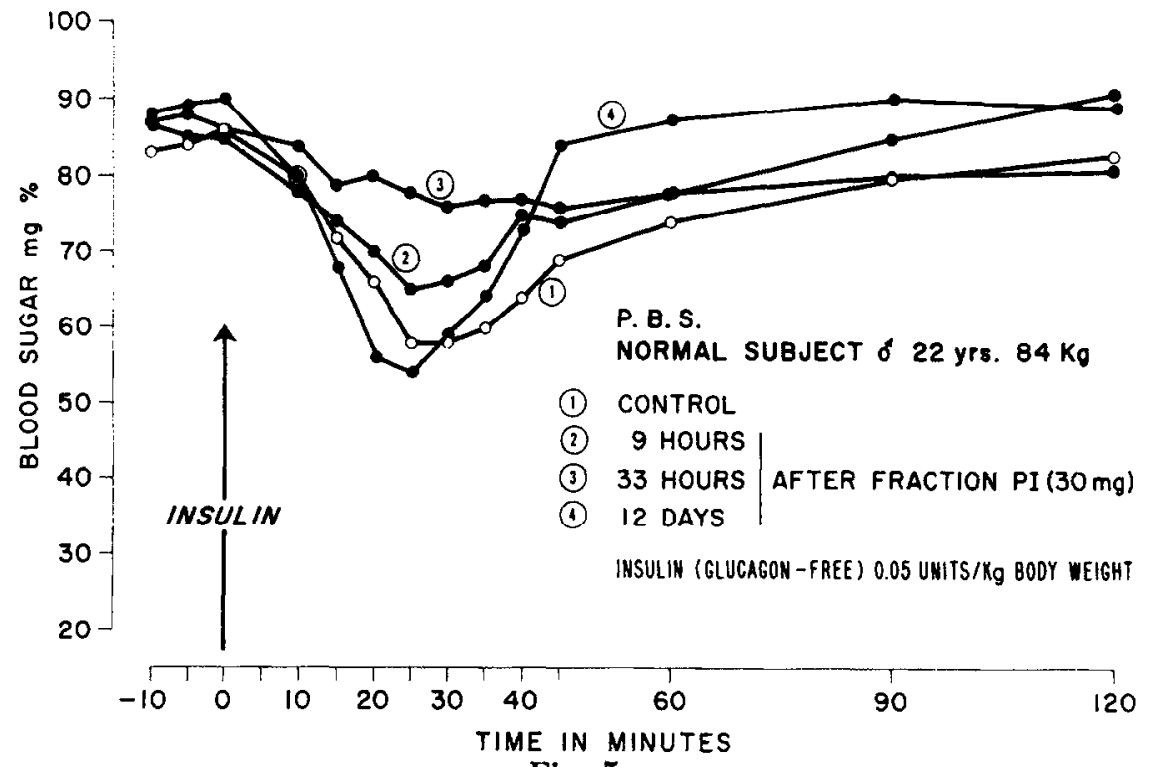

Fig. 5 


\section{PREPARATION OF FRACTION P III C 2}

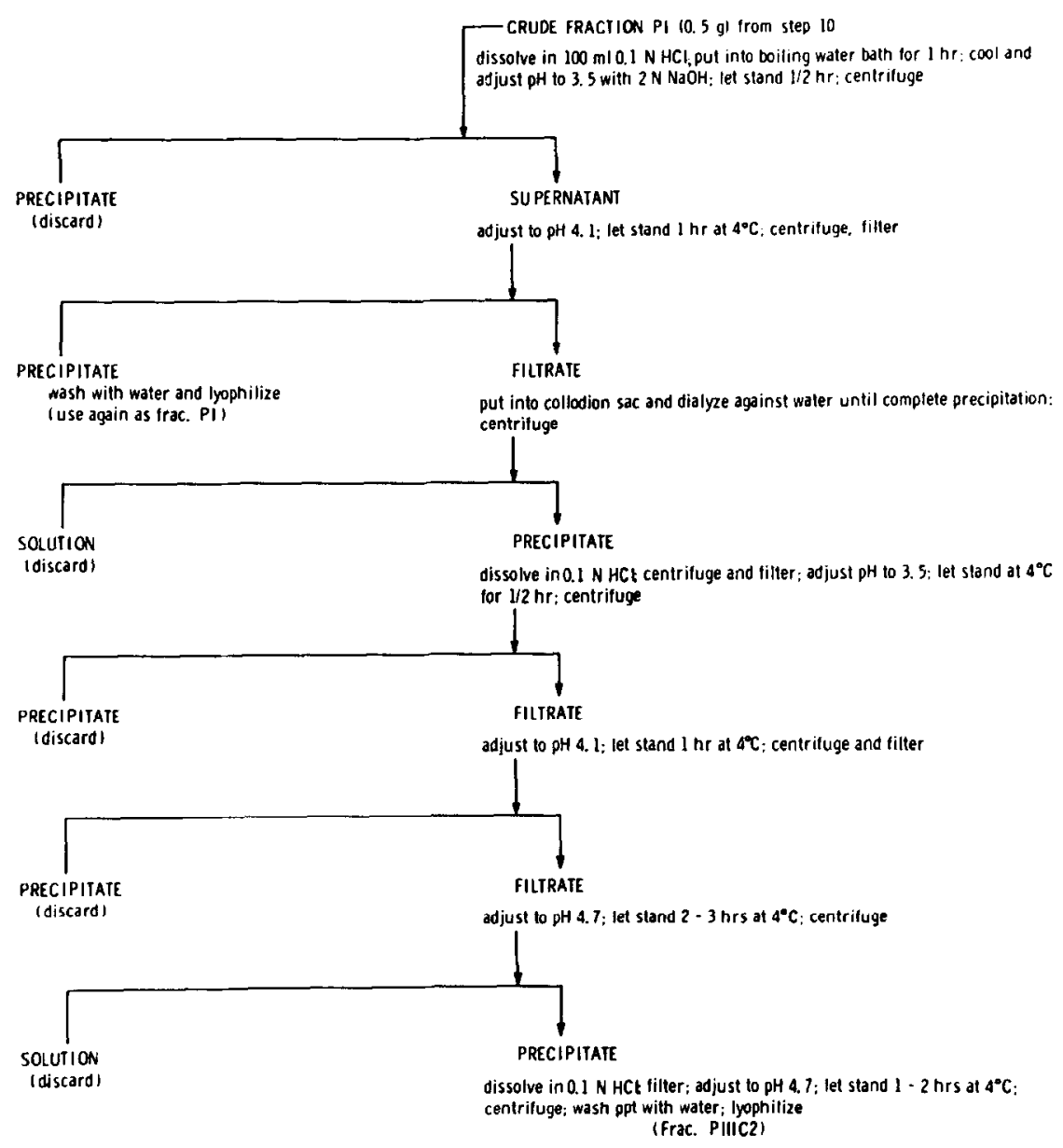

Fig. 6

The material is not bovine corticotropin since no evidence of adrenal cortical stimulation was observed. Pure bovine growth hormone does not diminish carbohydrate tolerance of man. ${ }^{8}$ It has been shown that bovine growth hormone can be altered by chemical manipulation after which it may induce carbohydrate intolerance in man. ${ }^{9}$ While this possibility exists, it seems an unlikely one since the isolation procedure employed in our work for the isolation of fraction PI is sufficiently mild that one would not anticipate disruption of the growth hormone molecule." Furthermore, the prolonged reduction in

${ }^{\circ}$ Dr. Don S. Shalch, School of Medicine and Dentistry of the University of Rochester showed that our fraction PI has brought about one thousandth the affinity for antigrowth hormone antibody as BGH. 


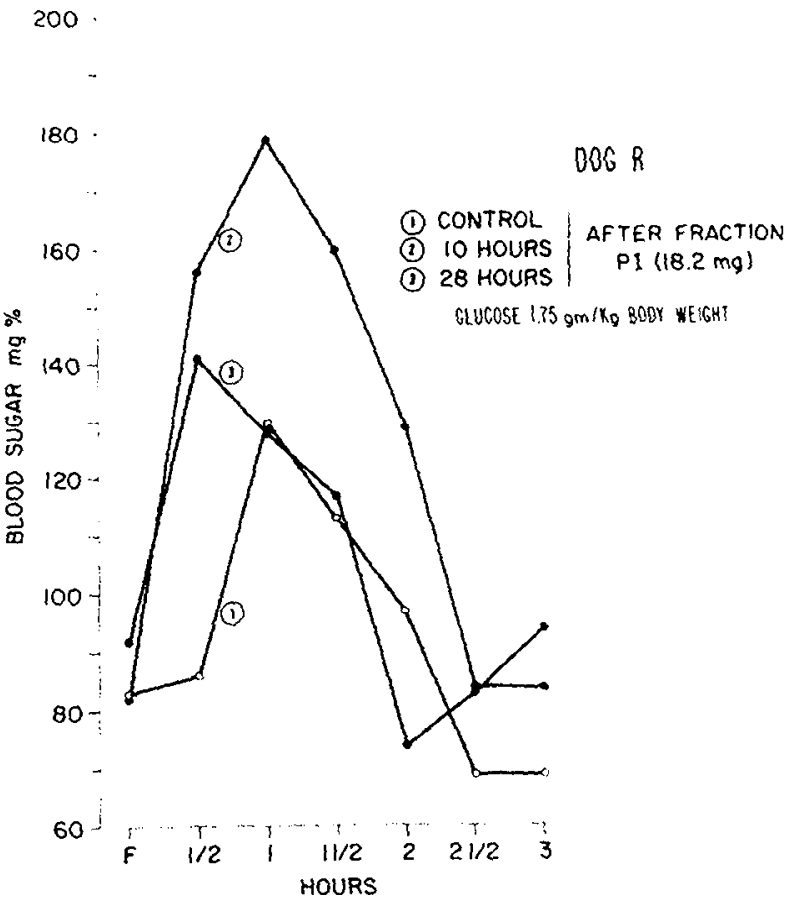

Fig. 7



Fig. 8 


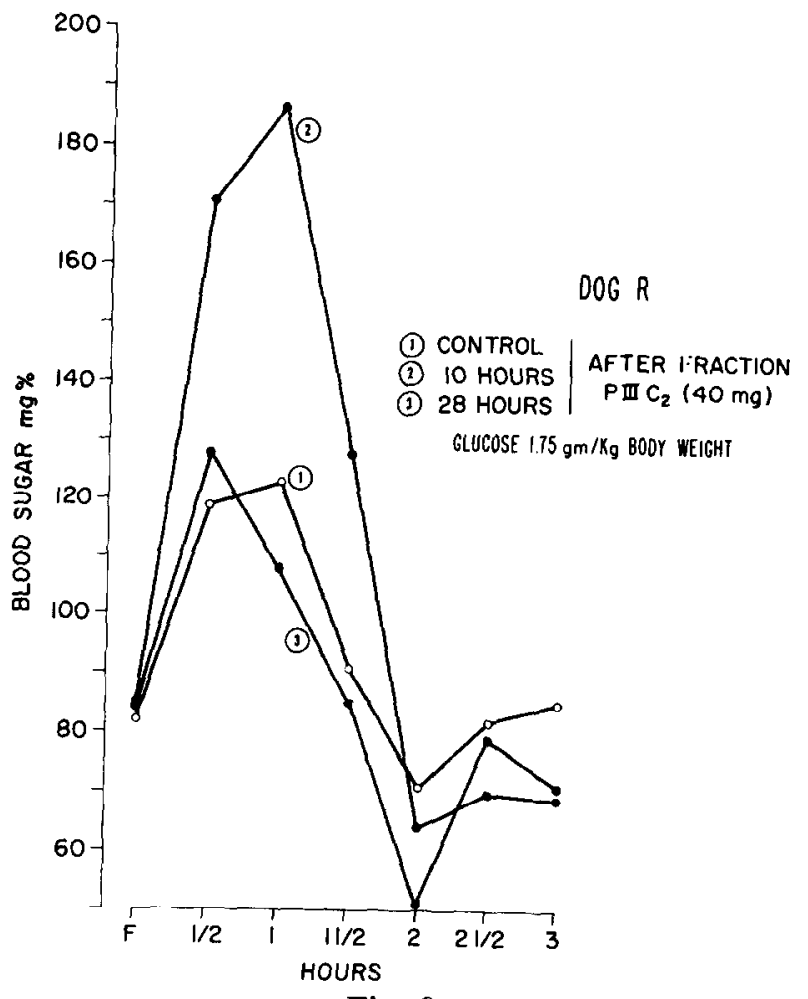

Fig. 9

V. M. B.

HORMAL SUBJECT \& $72 \mathrm{Kg}_{2} 21$ YRS

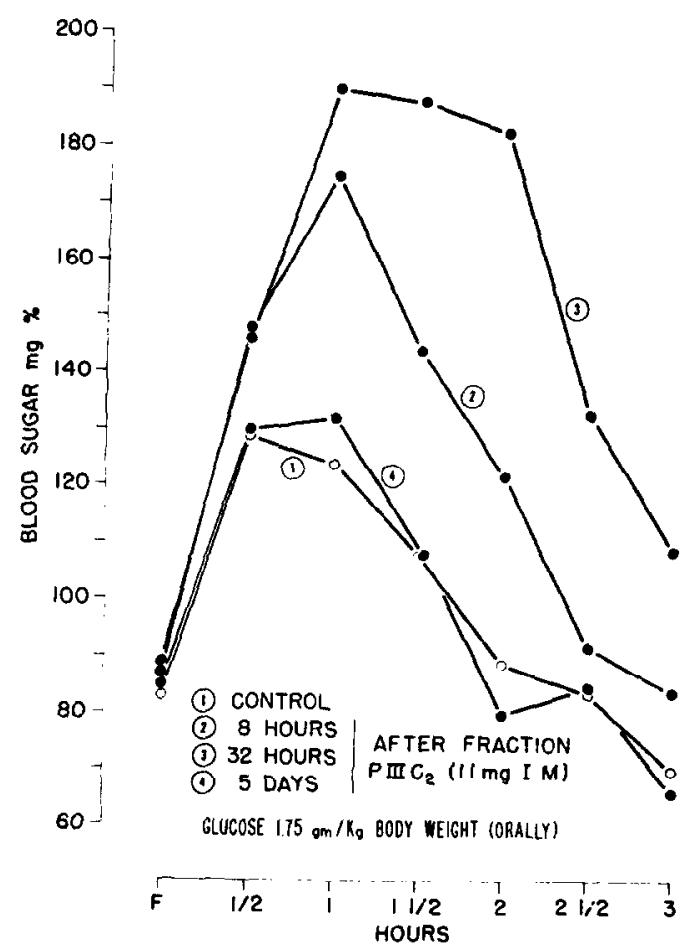

Fig. 10 
J.S.S.

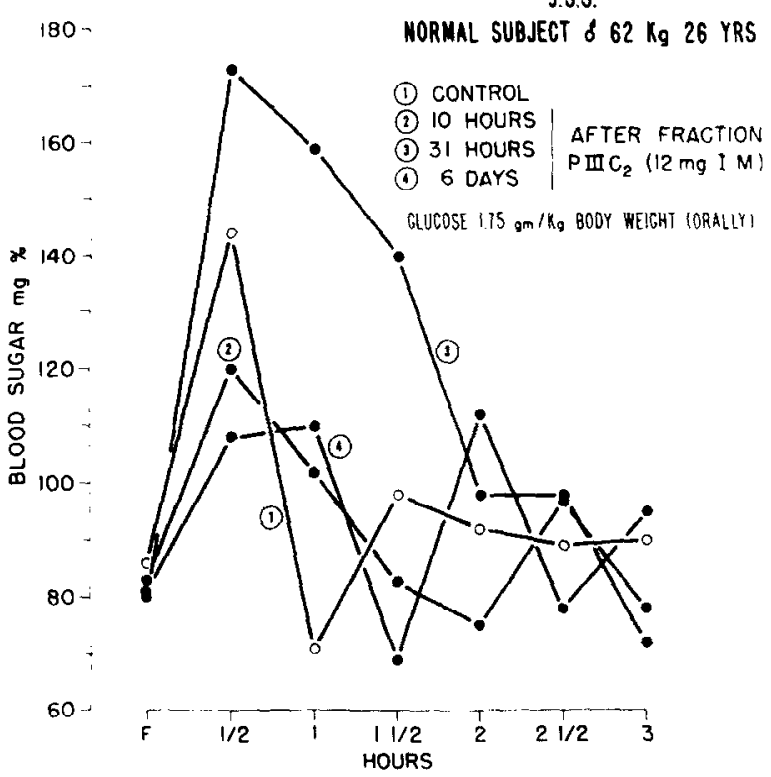

Fig. 11

Table 5.-Effect of Fraction PIIC2 upon Urinary 17-OHCS, 17-KS, Creatinine, Uric Acid and Glucose

\begin{tabular}{|c|c|c|c|c|c|c|c|}
\hline SUBJECT & DAY & INJECTION & $\begin{array}{c}17-O H C S \\
\text { mg/day }\end{array}$ & $\begin{array}{l}17-\mathrm{kS} \\
\mathrm{mg} / \mathrm{doy}\end{array}$ & $\begin{array}{c}\text { CREATININE } \\
\text { g/doy }\end{array}$ & $\begin{array}{l}\text { URIC ACID } \\
g / \text { doy }\end{array}$ & $\begin{array}{c}\text { GLUCOSE } \\
g / d 0 y\end{array}$ \\
\hline \multirow[b]{3}{*}{ W.M.B. } & $\begin{array}{l}1 \\
2\end{array}$ & $\begin{array}{l}0 \\
0\end{array}$ & $\begin{array}{l}5.6 \\
6.0\end{array}$ & $\begin{array}{l}6.2 \\
11.0\end{array}$ & $\begin{array}{l}1.68 \\
1.87\end{array}$ & $\begin{array}{l}0.834 \\
0.795\end{array}$ & $\begin{array}{l}1.16 \\
1.32\end{array}$ \\
\hline & 3 & $11 \mathrm{mg}(\mathrm{MM})$ & 6.0 & 8.2 & 1.83 & 0.773 & 1.24 \\
\hline & $\begin{array}{l}4 \\
5 \\
6 \\
7\end{array}$ & $\begin{array}{l}0 \\
0 \\
0 \\
0\end{array}$ & $\begin{array}{l}5.4 \\
5.2 \\
6.2 \\
6.4\end{array}$ & $\begin{array}{l}7.3 \\
5.7 \\
8.9 \\
9.3\end{array}$ & $\begin{array}{l}1.81 \\
1.73\end{array}$ & $\begin{array}{l}0.729 \\
0.385\end{array}$ & $\begin{array}{l}1.28 \\
1.40 \\
1.36 \\
1.28\end{array}$ \\
\hline \multirow{3}{*}{ J.S.S. } & $\begin{array}{l}1 \\
2\end{array}$ & $\begin{array}{l}0 \\
0\end{array}$ & $\begin{array}{l}6.0 \\
7.1\end{array}$ & $\begin{array}{l}12.8 \\
10.9\end{array}$ & $\begin{array}{l}1.59 \\
1.85\end{array}$ & $\begin{array}{l}0.742 \\
0.838\end{array}$ & $\begin{array}{l}0.750 \\
0.905\end{array}$ \\
\hline & 3 & $12 \mathrm{mg}(\mathrm{M})$ & 6.2 & 13.4 & 1.87 & 0.671 & 0.900 \\
\hline & $\begin{array}{l}4 \\
5\end{array}$ & $\begin{array}{l}0 \\
0\end{array}$ & $\begin{array}{l}8.2 \\
6.3\end{array}$ & $\begin{array}{l}16.2 \\
14.4 \\
\end{array}$ & $\begin{array}{l}2.27 \\
2.10\end{array}$ & $\begin{array}{l}1.047 \\
0.903\end{array}$ & $\begin{array}{l}1.280 \\
0.987\end{array}$ \\
\hline \multirow{3}{*}{ J.G.E. } & $\begin{array}{l}1 \\
2 \\
3\end{array}$ & $\begin{array}{l}0 \\
0 \\
0\end{array}$ & $\begin{array}{l}8.4 \\
8.4 \\
7.0\end{array}$ & $\begin{array}{l}19.2 \\
13.8 \\
18.2\end{array}$ & $\begin{array}{l}2.40 \\
1.85 \\
1.94\end{array}$ & $\begin{array}{l}1.009 \\
0.761 \\
0.803\end{array}$ & $\begin{array}{l}1.348 \\
1.240 \\
0.960\end{array}$ \\
\hline & 4 & $12 \mathrm{mq}$ (IM) & 8.2 & 16.4 & 2.36 & 1.042 & 1.360 \\
\hline & $\begin{array}{l}5 \\
6\end{array}$ & $\begin{array}{l}0 \\
0\end{array}$ & $\begin{array}{l}8.2 \\
9.2\end{array}$ & $\begin{array}{l}15.8 \\
17.8\end{array}$ & $\begin{array}{l}2.48 \\
2.23\end{array}$ & $\begin{array}{l}0.944 \\
0.868\end{array}$ & $\begin{array}{l}1.400 \\
1.400\end{array}$ \\
\hline
\end{tabular}


Table 6.-Effect of Fraction PIIIC2 upon Glucose Tolerance on a Normal Subject J. C. M. (22 years, male, $75 \mathrm{Kg}$.

\begin{tabular}{|c|c|c|c|c|c|c|c|}
\hline $\begin{array}{l}\text { HOURS AFTER } \\
\text { INJECTION } 40 \mathrm{mg}\end{array}$ & $F$ & $1 / 2$ & 1 & $11 / 2$ & 2 & $21 / 2$ & 3 \\
\hline Control & 83 & 147 & 134 & 102 & 101 & 110 & 88 \\
\hline 8 & 79 & 151 & $\begin{array}{c}152 \\
\text { (trace) }\end{array}$ & $\begin{array}{c}126 \\
\text { (trace) }\end{array}$ & 115 & 106 & 94 \\
\hline 32 & 83 & 137 & 165 & 134 & 111 & 114 & 106 \\
\hline 55 & 74 & 150 & $\begin{array}{c}158 \\
\text { (trace) }\end{array}$ & 130 & 125 & 123 & 100 \\
\hline 64 & 80 & 148 & $\begin{array}{c}188 \\
\text { (trace) }\end{array}$ & $\begin{array}{l}189 \\
1+1\end{array}$ & $\begin{array}{c}164 \\
\text { (trace) }\end{array}$ & 132 & 116 \\
\hline 78 & 74 & 151 & 158 & 137 & 120 & 110 & 105 \\
\hline 102 & 75 & 141 & $\begin{array}{l}185 \\
(+)\end{array}$ & $\begin{array}{l}130 \\
1+1\end{array}$ & $\begin{array}{c}108 \\
\text { (trace) }\end{array}$ & 113 & 89 \\
\hline 126 & 76 & 120 & 139 & 106 & 91 & 87 & 100 \\
\hline
\end{tabular}

Table 7.-Effect of Fraction PIIIC2 upon Glucose Tolerance on a Normal Subject R. F. J. (22 years, male, 59 Kg.)

\begin{tabular}{cccccccc}
\hline $\begin{array}{c}\text { HOURS AFTER } \\
\text { INJECTION } 40 \mathrm{mg}\end{array}$ & $F$ & $1 / 2$ & 1 & 1112 & 2 & $21 / 2$ & 3 \\
\hline Control & 76 & 121 & 101 & 72 & 92 & 105 & 87 \\
14 & 78 & 98 & 117 & 79 & 78 & 89 & 83 \\
38 & 80 & 128 & 133 & 83 & 98 & 90 & 75 \\
62 & 75 & 122 & $\begin{array}{c}110 \\
(\text { trace) }\end{array}$ & 71 & 67 & 74 & 71 \\
86 & 83 & 126 & $\begin{array}{c}134 \\
(\text { trace) }\end{array}$ & 106 & 78 & 76 & 77 \\
110 & 75 & 102 & 94 & 14 & 68 & 94 & 79 \\
134 & 76 & 129 & 143 & 101 & 77 & 83 & 89 \\
142 & 91 & 147 & 195 & 222 & 210 & 158 & 109 \\
158 & 69 & 108 & 144 & 77 & 101 & 92 & 75 \\
3 & & 109 & 95 & 87 & 82 & 88 & 93 \\
\hline
\end{tabular}


Table 8.-Effect of Fraction PIIIC2 upon Glucose Tolerance on a Normal Subject J. S. B. (22 years, male, $79 \mathrm{Kg}$.)

\begin{tabular}{cccccccc}
\hline $\begin{array}{c}\text { HOURS AFTER } \\
\text { INJECTION } 40 \mathrm{mg}\end{array}$ & $F$ & $1 / 2$ & 1 & $11 / 2$ & 2 & $21 / 2$ & 3 \\
\hline Control & 84 & 167 & 131 & 112 & 118 & 68 & 84 \\
12 & 87 & 183 & 162 & 144 & 115 & 84 & 91 \\
36 & 82 & 172 & 138 & 102 & 107 & 65 & 93 \\
60 & 76 & 162 & 131 & 72 & 118 & 53 & 57 \\
84 & 78 & 168 & 119 & 87 & 65 & 61 & 65 \\
108 & 81 & 168 & 129 & 99 & 74 & 78 & 52 \\
116 & 94 & 145 & 179 & 118 & 110 & 93 & 57 \\
135 & 78 & 190 & 192 & 157 & 134 & 77 & 110 \\
160 & 89 & 219 & 203 & 118 & 116 & 71 & 83 \\
184 & 82 & 211 & 126 & 150 & 85 & 86 & 73 \\
\hline
\end{tabular}

Table 9.- Effect of Fraction PIIIC2 upon Glucose Tolerance on a Normal Subject L. J. B. (22 years, male, $68 \mathrm{Kg}$.)

\begin{tabular}{|c|c|c|c|c|c|c|c|}
\hline $\begin{array}{l}\text { HOURS AFTER } \\
\text { INJECTION } 40 \mathrm{mg}\end{array}$ & $F$ & $1 / 2$ & I & $11 / 2$ & 2 & $21 / 2$ & 3 \\
\hline Control & 84 & 156 & 129 & 132 & 106 & 108 & 101 \\
\hline 14 & 83 & 148 & $\begin{array}{l}142 \\
1+1\end{array}$ & $\begin{array}{l}114 \\
1+1\end{array}$ & $\begin{array}{c}92 \\
\text { (trace) }\end{array}$ & 95 & 104 \\
\hline 36 & 84 & 153 & 168 & $\begin{array}{c}172 \\
(t++)\end{array}$ & $\begin{array}{l}140 \\
(++)\end{array}$ & $\begin{array}{c}125 \\
\text { (trace) }\end{array}$ & 86 \\
\hline 60 & 86 & 178 & $\begin{array}{l}190 \\
(++)\end{array}$ & $\begin{array}{c}162 \\
(+++)\end{array}$ & $\begin{array}{l}140 \\
(++)\end{array}$ & $\begin{array}{r}94 \\
1+1\end{array}$ & $\begin{array}{c}77 \\
\text { (trace) }\end{array}$ \\
\hline 84 & 79 & 149 & $\begin{array}{c}144 \\
\text { (trace) }\end{array}$ & $\begin{array}{c}132 \\
\text { (trace) }\end{array}$ & $\begin{array}{c}90 \\
\text { (trace) }\end{array}$ & 121 & 121 \\
\hline 106 & 83 & 148 & $\begin{array}{c}155 \\
\text { (trace) }\end{array}$ & $\begin{array}{c}142 \\
\text { (trace) }\end{array}$ & $\begin{array}{c}134 \\
\text { (trace) }\end{array}$ & $\begin{array}{c}99 \\
\text { (trace) }\end{array}$ & 107 \\
\hline 130 & 79 & 147 & $\begin{array}{l}131 \\
1+1\end{array}$ & $\begin{array}{c}115 \\
\text { (trace) }\end{array}$ & 105 & 101 & 101 \\
\hline 156 & 79 & 132 & 122 & 108 & 80 & 99 & 80 \\
\hline 228 & 86 & 152 & 112 & 133 & 88 & 93 & 98 \\
\hline
\end{tabular}




\section{W.M.B.}

NORMAL SUBJECT O $72 K_{g}$ 2I YRS.

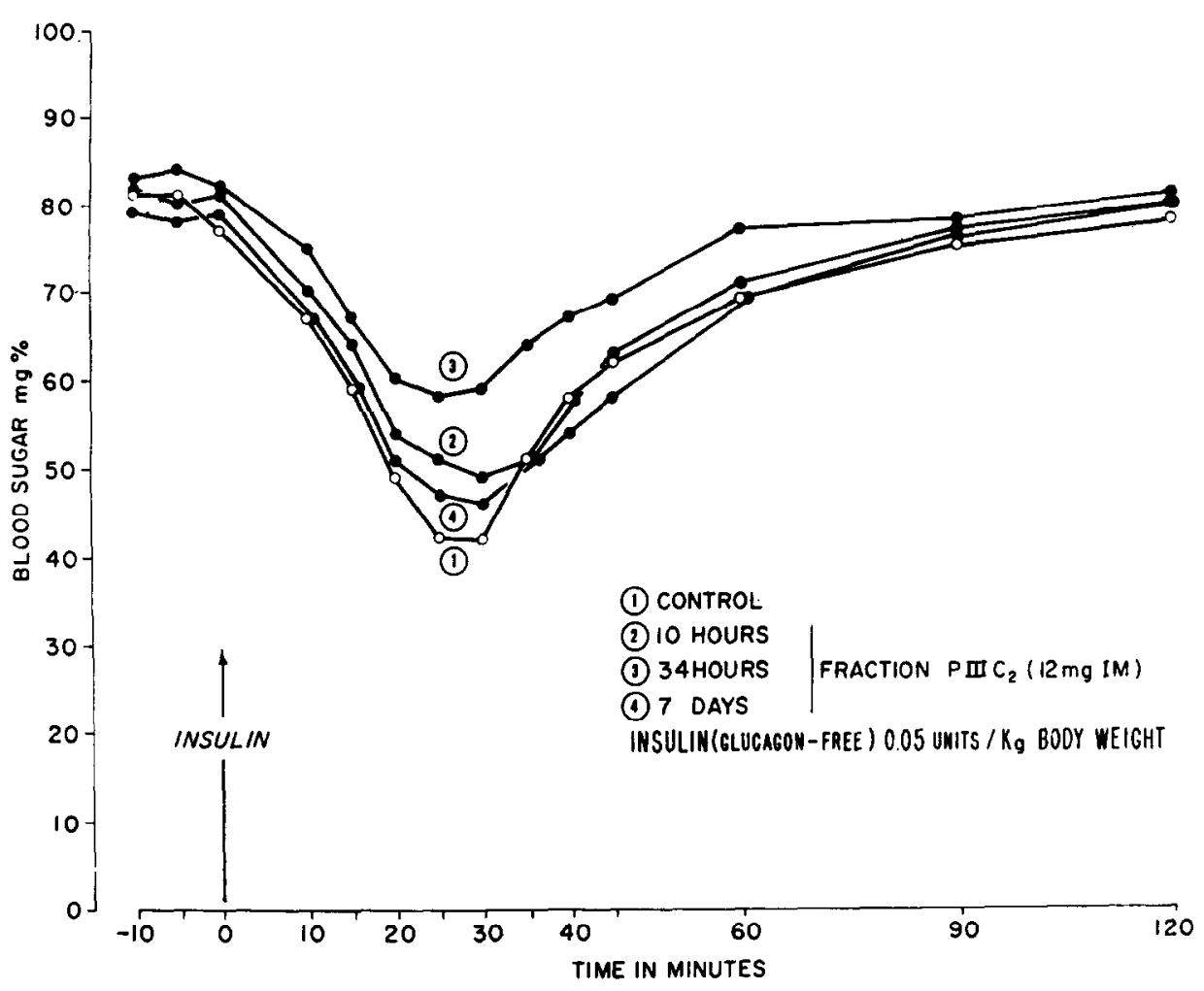

Fig. 12

carbohydrate tolerance induced by fraction PIIIC2 should have been accompanied by a rise of plasma free fatty acids and a fall of plasma amino nitrogen had the former been the result of a growth hormone effect.

It thus seems likely that the diabetogenic polypeptide isolated from the urine of patients with lipoatrophic diabetes is of adenohypophyseal origin and that it is different from the other anterior pituitary polypeptides known to diminish carbohydrate tolerance in man.

\section{REFERENCES}

1. Louis, L. H., and Minick. M. C.: Isolation of an insulin antagonist from urine of patients with lipoatrophic diabetes. J. Lab. Clin. Med. 60:995-996, 1962.

2. - Conn. J. W., and Minick, M. C.: Lipoatrophic diabetes: isolation and characterization of an insulin antagon- ist from urine. Metabolism 12:867886. 1963.

3. Chaney, A. L.: 17-ketosteroids in urine. Standard methods Clin. Chem. 2:79 85,1958 .

4. Silber, R. H., and Porter, C. C.: The determination of 17, 21-dihydroxy-20ketosteroids in urine and plasma. J. 
Biol. Chem. 210:923-932, 1954.

5. Forsham, P. H., Thorn, G. W., Prunty, F. T. Q., and Hills, A. G.: Clinical studies with pituitary adrenocorticotropin. J. Clin. Endocrinol. 8:15-66, 1948.

6. Somogyi, M.: Notes on sugar determination. J. Biol. Chem. 195:19, 1952.

7. Kingsley, G. R., and Schaffert, R. R.: Creatinine. Standard Methods Clin. Chem. 1:55-59, 1953.

8. Lewis, R. A., Klein, R., and Wilkins, L.:
The effect of pituitary growth hormone in dwarfism with osseous retardation and hypoglucemia and in a cretin treated with thyroid. J. Clin. Invest. 29:460-464, 1950.

9. Sonenberg, M., Free, C. A., Dellacha, J. M. Bonadonna, G.. Haymowitz, A. and Nadler, A. C.: The metabolic effects in man of bovine growth hormone digested with trypsin. J. Clin. Invest. 44:1099, 1965. 